\title{
ERODIBILIDADE DO SOLO NOS TABULEIROS COSTEIROS ${ }^{1}$
}

\author{
Sérgio Gualberto Martins ${ }^{2}$, Junior Cesar Avanzi ${ }^{3}$, \\ Marx Leandro Naves Silva ${ }^{4}$, Nilton Curi ${ }^{4}$, Sebastião Fonseca ${ }^{5}$
}

\begin{abstract}
SOIL ERODIBILITY IN THE BRAZILIAN COASTAL PLAINS

In order to determine soil losses caused by water erosion, in different situations, erosion prediction models, such as the Universal Soil Loss Equation (USLE), are used. Their application on agricultural and environmental planning depends on the determination of the USLE factors, including erodibility $(\mathrm{K}$ factor). The objective of this study was to determine erodibility for the main soil classes of the Brazilian Coastal Plains region, in Aracruz, Espírito Santo State. The experiment was established in the following soils: medium/clayey texture Yellow Argisol (PA1), Haplic Plinthosol (FX), and moderately rocky Yellow Argisol (PA2). For the calculation of soil erodibility, data of erosivity and soil losses, from November 1997 to May 2004, were used. Soil losses samplings were performed for each rainfall event regarded as erosive. The erodibility values were $0.007 \mathrm{Mg} \mathrm{h} \mathrm{MJ}^{-1} \mathrm{~mm}^{-1}$; $0.017 \mathrm{Mg} \mathrm{h} \mathrm{MJ}^{-1} \mathrm{~mm}^{-1}$; and $0.0004 \mathrm{Mg} \mathrm{h} \mathrm{MJ}^{-1} \mathrm{~mm}^{-1}$, for PA1, FX, and PA2, respectively.
\end{abstract}

KEY-WORDS: K factor; soil attributes; cohesive soils.

\section{INTRODUÇÃO}

A Equação Universal de Perdas de Solo (EUPS) é um importante instrumento na predição das perdas de solo e no planejamento do controle da erosão hídrica. A EUPS foi desenvolvida nos Estados Unidos, por Wischmeier \& Smith $(1965,1978)$, e tem sido utilizada em vários países. A EUPS, segundo Wischmeier \& Smith (1978), é composta pelos seguintes fatores: perda de solo (fator A - expresso em $\mathrm{Mg} \mathrm{ha}^{-1} \mathrm{ano}^{-1}$ ); erosividade da chuva (fator $\mathrm{R}$ - expresso em MJ mm ha ${ }^{-1} \mathrm{~h}^{-1}$ ano $^{-1}$ ); erodibilidade do solo (fator $\mathrm{K}$ - expresso em ha $\mathrm{Mg} \mathrm{h} \mathrm{ha} \mathrm{MJ}^{-1} \mathrm{MJ}^{-1} \mathrm{~mm}^{-1}$ ); fator topográfico (fator LS - adimensional), que considera a declividade (D - expresso em \%) e o comprimento de rampa do solo ( $\mathrm{L}$ - expresso em $\mathrm{m}$ ); índice de

\section{RESUMO}

Para determinar perdas de solo por erosão hídrica, em diferentes situações, são utilizados modelos de predição de erosão, como a Equação Universal de Perdas de Solo (EUPS). A aplicação destes modelos, no planejamento agrícola e ambiental, depende da determinação dos fatores da EUPS, dentre estes a erodibilidade (fator K). Este estudo teve como objetivo determinar a erodibilidade, para as principais classes de solos da região dos Tabuleiros Costeiros, em Aracruz (ES). O experimento foi instalado nos seguintes solos: Argissolo Amarelo textura média/ argilosa (PA1), Plintossolo Háplico (FX) e Argissolo Amarelo moderadamente rochoso (PA2). Para o cálculo da erodibilidade, foram utilizados dados de erosividade e de perdas de solo de novembro de 1997 a maio de 2004. As coletas de perdas de solo foram realizadas para cada evento de chuva considerada erosiva. Os valores de erodibilidade foram $0,007 \mathrm{Mg} \mathrm{h} \mathrm{MJ}^{-1} \mathrm{~mm}^{-1}$; $0,017 \mathrm{Mg} \mathrm{h} \mathrm{MJ}^{-1} \mathrm{~mm}^{-1}$; e $0,0004 \mathrm{Mg} \mathrm{h} \mathrm{MJ}^{-1} \mathrm{~mm}^{-1}$, para PA1, FX e PA2, respectivamente.

PALAVRAS-CHAVE: Fator K; atributos de solo; solos coesos.

cobertura vegetal (fator C - adimensional); e práticas conservacionistas (fator $\mathrm{P}$ - adimensional), sendo que, em muitos casos, o fator $\mathrm{C}$ pode ser determinado em associação com o fator $\mathrm{P}(\mathrm{CP})$.

Na EUPS, a erodibilidade consiste no fator responsável pela susceptibilidade do solo à erosão hídrica, sendo condicionada, basicamente, por atributos mineralógicos, químicos, morfológicos, físicos e biológicos do solo (Dumas 1965, Wischmeier \& Mannering 1969, El-Swaify \& Dangler 1977). A erodibilidade representa o efeito dos processos que regulam a infiltração da água no solo, a desagregação pelo impacto da gota de chuva e a resistência ao transporte pelo fluxo superficial, os quais são responsáveis pelo comportamento do solo, em relação aos processos erosivos (Lal 1988). O valor de erodibilidade é mui-

1. Trabalho recebido em abr./2010 e aceito para publicação em ago./2011 (n ${ }^{\circ}$ registro: PAT 9604/ DOI: 10.5216/pat.v41i3.9604).

2. Centro Universitário de Lavras, Lavras, MG, Brasil.E-mail: sergio.gualberto@unilavras.edu.br.

3. Embrapa Pesca e Aquicultura, Palmas, TO, Brasil.E-mail: junior.avanzi@embrapa.br.

4. Universidade Federal de Lavras, Departamento de Ciência do Solo, Lavras, MG, Brasil.E-mails: marx@dcs.ufla.br, niltcuri@dcs.ufla.br. 5. Fibria Celulose S. A., Aracruz, ES, Brasil. E-mail: sf@fibria.com.br. 
to variável, devido à ampla variedade de solos com atributos diferenciados, tornando arriscado estimar um valor, com base, unicamente, na classificação do solo (El-Swaify \& Dangler 1982, Silva et al. 1997).

No Brasil, um número considerável de solos já tem o seu valor de erodibilidade determinado com o uso de simuladores de chuva e, também, sob chuva natural (Marques et al. 1997a). Conforme levantamento realizado por Denardin (1990) e Silva et al. (2000, 2009), a erodibilidade de solos com horizonte B latossólico varia $0,002-0,034 \mathrm{Mg} \mathrm{h} \mathrm{MJ}^{-1} \mathrm{~mm}^{-1}$, enquanto os solos que possuem horizonte B textural (argissolos, luvissolos, B nítico e B plânico) variam 0,004-0,045 Mg h MJ'-1 $\mathrm{mm}^{-1}$ (Denardin 1990, Marques et al. 1997a e 1997b). Para os cambissolos, os valores encontrados foram $0,011 \mathrm{Mg} \mathrm{h} \mathrm{MJ}^{-1} \mathrm{~mm}^{-1}$ (Bertol et al. 2002) e $0,026 \mathrm{Mg} \mathrm{h} \mathrm{MJ}^{-1} \mathrm{~mm}^{-1}$ (Silva et al. 2009).

A determinação direta do fator $\mathrm{K}$ é feita por meio da instalação de parcelas de perdas de solo no campo, seja sob chuva natural ou simulada, as quais envolvem altos custos, além de demandar vários anos de coleta de dados (Silva et al. 1999). Laflen (1982) sugere que o tempo mínimo de condução do experimento a campo seja de cinco anos, evitando-se, assim, a variabilidade da erodibilidade. Por este motivo, vários pesquisadores têm proposto a determinação do fator K de maneira indireta. Silva et al. (2000), entretanto, testaram 23 modelos de estimativa da erodibilidade para latossolos, sendo que todos os modelos testados foram inadequados para tal estimativa. Da mesma forma, a avaliação de treze modelos indiretos de avaliação do fator $\mathrm{K}$, em solos com horizontes $\mathrm{B}$ textural, mostrou que nenhum dos métodos foi recomendado para estimar a erodibilidade do solo (Marques et al. 1997b). Pelos trabalhos apresentados, sugere-se que a determinação deste fator, para os solos brasileiros, seja realizada por meio de experimentos de campo, evitando-se possíveis erros causados pelo uso de metodologias desenvolvidas para outras regiões.

Com base no exposto, este estudo teve como objetivo determinar, de maneira direta, a erodibilidade (fator K), para as principais classes de solos representativas da região dos Tabuleiros Costeiros, no município de Aracruz (ES).

\section{MATERIAL E MÉTODOS}

O experimento para determinação da erodibilidade foi instalado nas principais classes de solos existentes na região dos Tabuleiros Costeiros do Espírito Santo, originados de sedimentos da Formação Barreiras, a saber: Argissolo Amarelo textura média/ argilosa (PA1), Plintossolo Háplico (FX) e Argissolo Amarelo moderadamente rochoso (PA2).

O clima da região é Aw, segundo a classificação de Köppen, com temperatura média de $23^{\circ} \mathrm{C}$ e precipitação média anual de $1.400 \mathrm{~mm}$ (Embrapa 2000). A região onde se insere a unidade de pesquisa está situada entre as coordenadas $19^{\circ} 35^{\prime}$ e $20^{\circ} 15^{\prime}$ de latitude Sul e $40^{\circ} 00^{\prime}$ e $40^{\circ} 20^{\prime}$ de longitude Oeste. No período de estudo, a precipitação anual média variou 788-1.342 $\mathrm{mm}$, dados obtidos por meio da estação climatológica automatizada localizada na área de estudo.

Para gerar o fator $\mathrm{K}$, foram utilizados dados de perdas de solo sob chuva natural, de novembro de 1997 a maio de 2004. A avaliação das perdas de solo foi realizada por meio de parcelas em área sem cobertura, segundo preconizado por Wischmeier \& Smith (1978). Entretanto, estas apresentaram algumas modificações (4 m x $12 \mathrm{~m}$ ), em relação ao tamanho recomendado para a parcela padrão. O maior comprimento obedeceu ao sentido do declive. As perdas de solo foram correlacionadas com a erosividade da chuva, determinada para o período de estudo.

Para o cálculo da erosividade, foram utilizados dados pluviométricos de chuva obtidos em estações climatológicas automatizadas, localizadas na área do experimento, que, para este estudo, geraram dados de cinco em cinco minutos. As informações de precipitações foram coletadas de novembro de 1997 a maio de 2004. A partir das precipitações, foram calculadas as energias cinéticas totais das chuvas, para cada evento. A energia cinética foi obtida de acordo com a equação $\mathrm{E}=0,119+0,0873 \operatorname{LogI}$, proposta por Wischmeier \& Smith (1958), em que E é a energia cinética (em MJ ha ${ }^{-1} \mathrm{~mm}^{-1}$ ) e I a intensidade da chuva $\left(\mathrm{em} \mathrm{mm} \mathrm{h}^{-1}\right)$. O índice $\mathrm{EI}_{30}$ foi obtido a partir da multiplicação da energia cinética total (E) de uma chuva erosiva pela máxima intensidade ocorrida em um período de 30 minutos consecutivos $\left(\mathrm{I}_{30}\right)$, segundo Wischmeier \& Smith (1958). As chuvas erosivas foram aquelas com precipitação pluvial superior a $10 \mathrm{~mm}$, ou com intensidade máxima, em 15 minutos, maior que $24 \mathrm{~mm} \mathrm{~h}^{-1}$, ou com energia cinética superior a 3,6 MJ (De Maria 1994). Pelo somatório dos valores do índice $\mathrm{EI}_{30}$, obtido em $\mathrm{MJ} \mathrm{mm} \mathrm{ha}^{-1} \mathrm{~h}^{-1}$, em cada mês, obteve-se o índice mensal e, somando-se os valores mensais, obteve-se o índice anual. 
Os valores de erodibilidade foram calculados por meio do quociente entre a perda média anual de solo $\left(\mathrm{Mg} \mathrm{ha}^{-1}\right)$ e a erosividade média anual das chuvas (MJ mm ha ${ }^{-1} \mathrm{~h}^{-1}$ ), seguindo-se o procedimento de Wischmeier \& Smith (1978), bem como por análise de regressão linear simples $(y=a+b x)$, utilizando-se as perdas de solo no eixo (y) e a erosividade da chuva no eixo (x), em que o coeficiente $b$, após correções do declive, representa a erodibilidade do solo, seguindo-se procedimento de Wischmeier \& Mannering (1969).

Para caracterização do solo, foram coletadas amostras dos horizontes A e B, das referidas classes de solo. Entretanto, as amostras foram coletadas em condições de mata nativa, garantindo-se as condições de solos não perturbados. Na fração TFSA (inferior a $2 \mathrm{~mm}$ ), foram feitas análises de carbono orgânico (Embrapa 1997). Foi realizada, também, análise granulométrica, utilizando-se o método da pipeta (Day 1965), com e sem o emprego de dispersante químico $(\mathrm{NaOH} 1 \mathrm{~N})$. A argila dispersa em água (ADA) foi determinada sem o uso de dispersante químico. O índice de floculação (IF) foi calculado de acordo com a Embrapa (1997).

A densidade do solo foi determinada em amostras com estrutura indeformada, coletadas com amostrador de Uhland (Grossman \& Reinsch 2002), e a densidade de partículas pelo método do balão volumétrico (Flint \& Flint 2002a). O volume total de poros foi determinado conforme Flint \& Flint (2002b). Agregados de diâmetro entre $7,93 \mathrm{~mm}$ e $4,76 \mathrm{~mm}$ foram submetidos, após pré-tratamento de umedecimento lento, ao tamisamento, por meio de um jogo de peneiras de $2 \mathrm{~mm} ; 1 \mathrm{~mm} ; 0,5 \mathrm{~mm} ; 0,25 \mathrm{~mm}$; e 0,105 mm (Nimmo \& Perkins 2002). Após determinação das proporções de agregados, em cada peneira, calculou-se o diâmetro médio geométrico (DMG). A permeabilidade do solo foi avaliada no campo, por meio da taxa constante de infiltração de água, a $15 \mathrm{~cm}$ de profundidade, com duas cargas constantes de $3 \mathrm{~cm}$ e $6 \mathrm{~cm}$ de coluna d'água, utilizando-se o permeâmetro de Guelph (Reynolds \& Elrick 1992). Na Tabela 1, são

Tabela 1. Atributos mineralógicos, químicos, físicos e morfológicos dos solos estudados, para condição de mata nativa (Aracruz, ES, 2000).

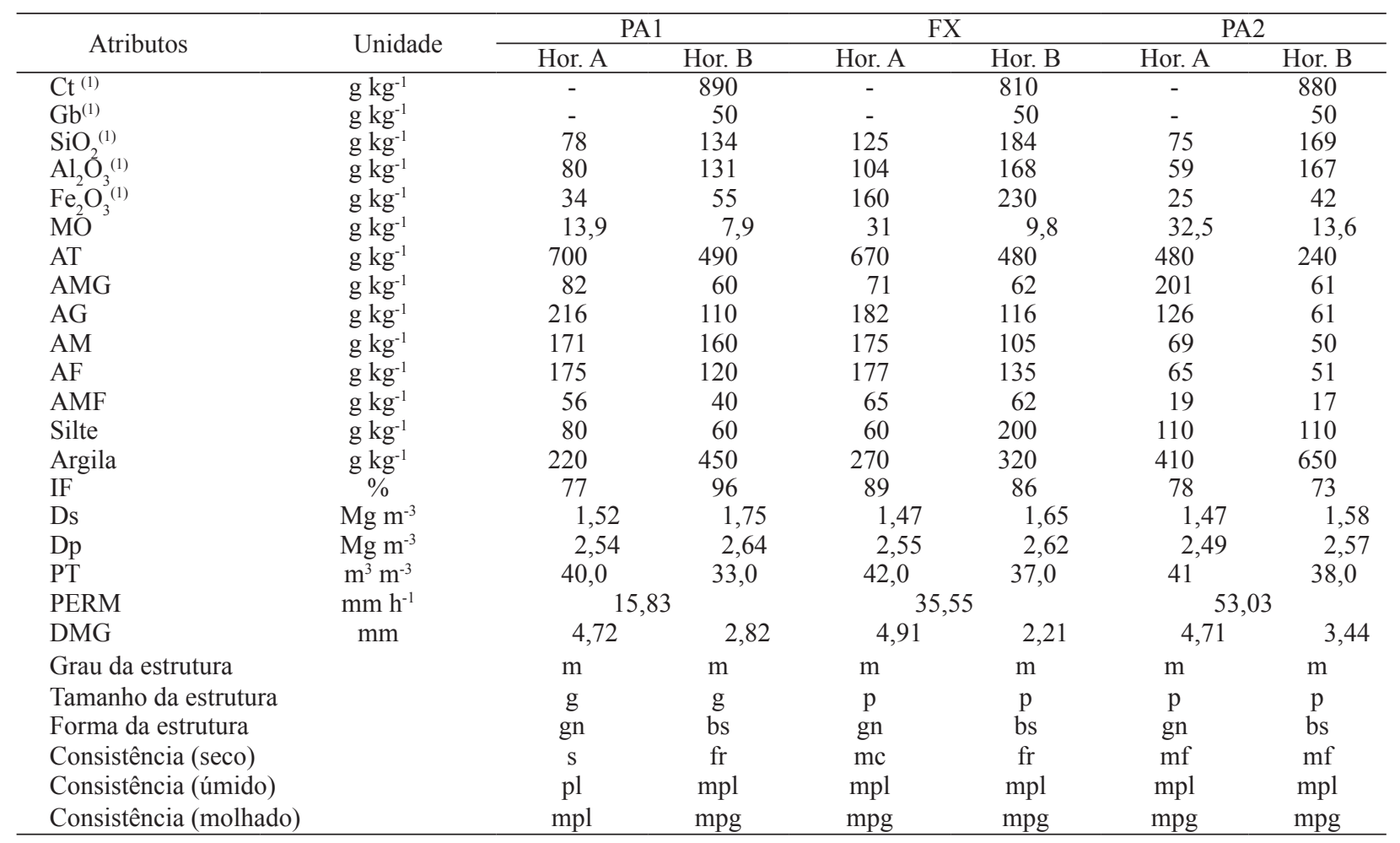

${ }^{(1)}$ Fonte: Duarte et al. (2000). PA1: Argissolo Amarelo textura média/argilosa; FX: Plintossolo Háplico; PA2: Argissolo Amarelo moderadamente rochoso; Ct: caulinita; Gb: gibbsita; MO: matéria orgânica; AT: areia total; AMG: areia muito grossa; AG: areia grossa; AM: areia média; AF: areia fina; AMF: areia muito fina; IF: índice de floculação; Ds: densidade do solo; Dp: densidade de partículas; PT: porosidade total; PERM: permeabilidade do solo à água; DMG: diâmetro médio geométrico; m: moderada; f: fraca; g: grande; p: pequena; gn: granular; bs: blocos subangulares; s: solto; fr: friável; mc: macio; mf: muito friável; pl: plástico; mpg: muito pegajoso; e mpl: muito plástico. 
apresentados os resultados dos atributos mineralógicos, químicos, físicos e morfológicos dos solos estudados.

\section{RESULTADOS E DISCUSSÃO}

$\mathrm{Na}$ determinação da erodibilidade, o coeficiente angular (b) das equações de regressão, obtidas por meio das variáveis erosividade, expressa pelo índice $\mathrm{EI}_{30}$, e perdas de solo (A), apresenta, segundo Wischmeier (1972), uma boa estimativa do fator erodibilidade. Neste estudo, foram avaliadas 1.024 ocorrências de chuvas, das quais 390 foram erosivas e 634 não erosivas. Partindo-se dos eventos de chuvas erosivas, determinou-se a equação linear para os solos em estudo. Assim, os coeficientes dos modelos lineares podem ser observados na Tabela 2.

Os modelos $\mathrm{A}=0,0015 \mathrm{EI}_{30}+0,0215\left(\mathrm{R}^{2}=\right.$ $0,96)$, para o PA1; $\mathrm{A}=0,0029 \mathrm{EI}_{30}-0,0518\left(\mathrm{R}^{2}=0,90\right)$, para o $\mathrm{FX} ; \mathrm{eA}=0,0027 \mathrm{EI}_{30}+0,6477\left(\mathrm{R}^{2}=0,98\right)$, para o $\mathrm{PA} 2$, consideraram valores anuais e foram os modelos que apresentaram os maiores coeficientes de determinação (Figura 1). Para condições climáticas de região temperada, Wischmeier (1959) encontrou coeficiente de determinação de 0,96 , entre perdas de solo e o $\mathrm{EI}_{30}$.

Os modelos lineares foram corrigidos para que as coordenadas iniciais partissem da origem, apresentando-se da seguinte forma: $\mathrm{P}=0,0015 \mathrm{EI}_{30}$ $\left(\mathrm{R}^{2}=0,93\right), \mathrm{P}=0,0026 \mathrm{EI}_{30}\left(\mathrm{R}^{2}=0,89\right)$ e $\mathrm{P}=0,0027$ $\mathrm{EI}_{30}\left(\mathrm{R}^{2}=0,951\right)$, para o PA1, FX e PA2, respectivamente. Para a determinação da erodibilidade, o coeficiente angular de cada equação foi corrigido pelo fator topográfico. O fator topográfico (LS) foi de 0,$23 ; 0,17$; e 6,45 , para o PA1, FX e PA2, respectivamente. $\mathrm{O}$ coeficiente linear $\mathrm{b}$, depois de corrigido pelo fator topográfico dos respectivos solos, permitiu obter os valores de erodibilidade dos solos, que foram estimados em $0,007 \mathrm{Mg} \mathrm{h} \mathrm{MJ}^{-1} \mathrm{~mm}^{-1}$; $0,017 \mathrm{Mg} \mathrm{h} \mathrm{MJ}^{-1} \mathrm{~mm}^{-1}$; e $0,0004 \mathrm{Mg} \mathrm{h} \mathrm{MJ}^{-1} \mathrm{~mm}^{-1}$, para o PA1, FX e PA2, respectivamente. Estes valores foram classificados como baixos, para o PA1 e PA2, e moderados, para o FX, segundo Foster et al. (1981). Os valores de erodibilidade encontrados neste estudo, com exceção do PA2, estão dentro da faixa de erodibilidade para os solos brasileiros com B textural, determinada por Denardin (1990) e Marques et al. (1997a).

Os valores de erodibilidade, classificados como baixo e moderado, obtidos para os solos estudados, estão relacionados à sua constituição mineralógica e química. Estes solos apresentam mi-
Tabela 2. Parâmetros das equações de regressão da forma $y=$ $\mathrm{a}+\mathrm{bx}$, entre os índices $\mathrm{EI}_{30}$ de erosividades anuais da chuva (x) e perdas de solo (y), e coeficientes de determinação $\left(\mathrm{R}^{2}\right)$, em condições de solo descoberto (Aracruz, ES, 2004).

\begin{tabular}{cccc}
\hline \multirow{2}{*}{ Solo } & \multicolumn{2}{c}{ Parâmetros da equação } & \multirow{2}{*}{$\begin{array}{c}\text { Coeficiente de } \\
\text { determinação }\left(\mathrm{R}^{2}\right)\end{array}$} \\
\cline { 2 - 3 } & $\mathrm{a}$ & $\mathrm{b}$ & $0,96^{* *}$ \\
PA1 & 0,0215 & 0,0015 & $0,90^{* *}$ \\
FX & $-0,0121$ & 0,0026 & $0,98^{* *}$ \\
PA2 & 0,6477 & 0,0027 & \\
\hline
\end{tabular}

PA1: Argissolo Amarelo textura média/argilosa; FX: Plintossolo Háplico; PA2: Argissolo Amarelo moderadamente rochoso.** Significativo a $0,01 \%$.
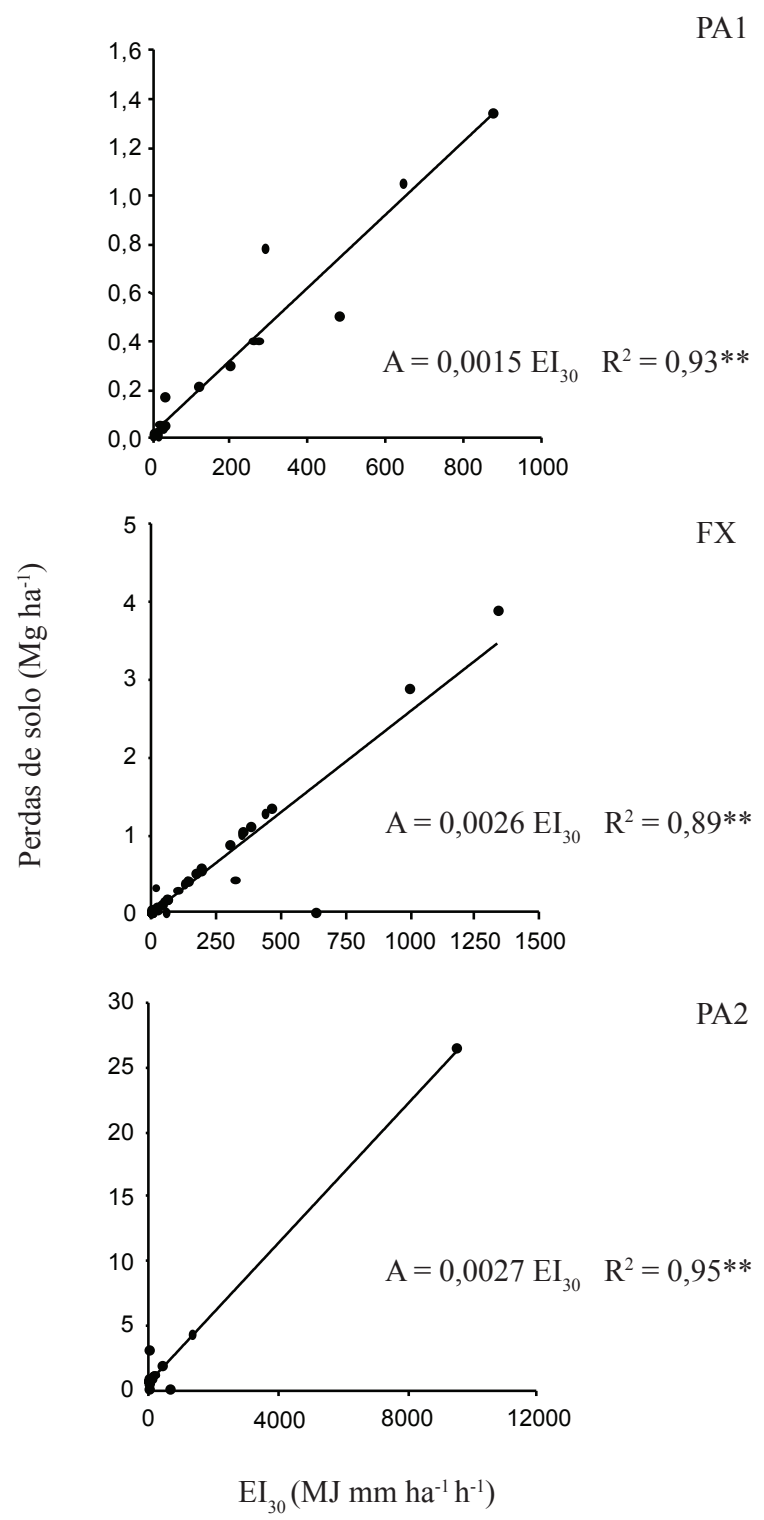

Figura 1. Relação entre perdas de solo e erosividade $\left(\mathrm{EI}_{30}\right)$, para os solos da região dos Tabuleiros Costeiros (Aracruz, ES, 2004). PA1: Argissolo Amarelo textura média/argilosa; FX: Plintossolo Háplico; PA2: Argissolo Amarelo moderadamente rochoso. $* *$ Significativo a $0,01 \%$. 
neralogia essencialmente caulinítica e baixos teores de óxidos de ferro (Tabela 1), contribuindo para uma alta coesão. Apesar da baixa permeabilidade do solo à água, estas forças de atração determinadas pela coesão dificultam a quebra de agregados provocada pelo impacto das gotas de chuva, o que aumenta a resistência destes solos à erosão hídrica.

Outros aspectos observados neste estudo foram os altos valores do diâmetro médio geométrico (DMG) e índice de floculação (Tabela 1), que, certamente, contribuíram para os valores baixos e moderados de erodibilidade dos solos estudados. De acordo com Ângulo et al. (1984), um dos parâmetros que melhor se correlacionam com a erodibilidade é a agregação do solo, determinada por meio da estabilidade de agregados em água, expressa pelo diâmetro médio geométrico (DMG). De acordo com estes autores, quanto menor o DMG, maior é a erodibilidade do solo. Embora os solos tenham apresentado valores baixos de permeabilidade (Tabela 1), o que poderia conferir a estes solos uma alta erodibilidade, o balanço dos outros atributos avaliados parece superar este aspecto, conferindo a estes solos baixos valores de erodibilidade.

\section{CONCLUSÃO}

Após sete anos de condução do experimento, determinaram-se os seguintes valores de erodibilidade para os solos da região dos Tabuleiros Costeiros: $0,007 \mathrm{Mg} \mathrm{h} \mathrm{MJ}{ }^{-1} \mathrm{~mm}^{-1}$, para Argissolo Amarelo textura média/argilosa; $0,017 \mathrm{Mg} \mathrm{h} \mathrm{MJ}^{-1} \mathrm{~mm}^{-1}$, para Plintossolo Háplico; e $0,0004 \mathrm{Mg} \mathrm{h} \mathrm{MJ}^{-1} \mathrm{~mm}^{-1}$, para Argissolo Amarelo moderadamente rochoso.

\section{REFERÊNCIAS}

ÂNGUlO, R. J.; ROLOFF, G.; SOUZA, M. L. P. Relações entre a erodibilidade e agregação, granulometria e características químicas de solos brasileiros. Revista Brasileira de Ciência do Solo, Viçosa, v. 8, n. 1, p. 133138, 1984.

BERTOL, I. et al. Erosividade das chuvas e sua distribuição entre 1989 e 1998 no município de Lages (SC). Revista Brasileira de Ciência do Solo, Viçosa, v. 26, n. 2, p. 455464, 2002.

DAY, P. R. Particle fractionation and particle-size analysis. In: BLACK, C. A. (Ed.). Methods of soil analysis. Madison: ASA, 1965. p. 545-566.
DE MARIA, I. C. Cálculo da erosividade da chuva. In: Manual de programas de processamento de dados de campo e de laboratório para fins de experimentação em conservação do solo. Campinas: IAC-SCS, 1994. Não paginado.

DENARDIN, J. R. Erodibilidade do solo estimada por meio de parâmetros fisicos e químicos. 1990. $106 \mathrm{f}$. Tese (Doutorado em Solos e Nutrição de Plantas)-Escola Superior de Agricultura Luiz de Queiroz, Piracicaba, 1990.

DUARTE, M. N. et al. Mineralogia, química e micromorfologia de solos de uma microbacia nos Tabuleiros Costeiros do Espírito Santo. Pesquisa Agropecuária Brasileira, Brasília, DF, v. 35, n. 6, p. 1237 1250 , jun. 2000.

DUMAS, J. Relation entre l'érodibilité des sols et leurs caractéristiques analytiques. Cahiers Orstom, Paris, v. 3, n. 4, p. 307-333, 1965.

El-SWAIFY, S. A.; DANGLER, E. W. Erodibilities of selected tropical soils in relation to structural and hydrologic parameters. In: NATIONAL CONFERENCE ON SOIL EROSION, 30., West Lafayette. Proceedings... Ankeny: Soil Conservation Society of America, 1977. p. 105-114.

El-SWAIFY, S. A.; DANGLER, E. W. Rainfall erosion in the tropics: a state of art. In: KREBS, E. D. Determinants of soil loss tolerance. Madison: American Society of Agronomy, 1982. p. 1-25.

EMPRESA BRASILEIRA DE PESQUISA AGROPECUÁRIA (Embrapa). Centro Nacional de Pesquisa de Solos. Levantamento generalizado e semidetalhado de solos da Aracruz Celulose S. A. no Estado do Espirito Santo e no extremo sul do Estado da Bahia e sua aplicação aos plantios de eucalipto. Rio de Janeiro: Embrapa, 2000.

EMPRESA BRASILEIRA DE PESQUISA AGROPECUÁRIA (Embrapa). Centro Nacional de Pesquisa de Solos. Manual de métodos de análise de solos. 2. ed. Rio de Janeiro: Embrapa, 1997.

FLINT, A. L.; FLINT, L. E. Particle density. In: DANE, J. H.; TOPP, G. C. (Eds.). Methods of soil analysis: physical methods. Madison: Soil Science Society of America, 2002a. p. 229-240.

FLINT, L. E.; FLINT, A. L. Porosity. In: DANE, J. H.; TOPP, G. C. (Eds.). Methods of soil analysis: physical methods. Madison: Soil Science Society of America, 2002b. p. 241-254.

FOSTER, G. R. et al. Conversion of the universal soil loss equation to SI metric units. Journal of Soil and Water Conservation, Ankeny, v. 36, n. 4, p. 355-359, 1981. 
GROSSMAN, R. B.; REINSCH, T. G. Bulk density and linear extensibility. In: DANE, J. H.; TOPP, G. C. (Eds.). Methods of soil analysis: physical methods. Madison: Soil Science Society of America, 2002. p. 201-228.

LAFLEN, J. M. Special problems of the USLE: soil erodibility (K). In: WORKSHOP ON ESTIMATING EROSION AND SEDIMENT YIELD ON RANGELANDS, 1., Tucson. Proceedings... Oakland: Agricultural Research Service, 1982. p. 63-72.

LAL, R. Erodibility and erosivity. In: LAL, R. (Ed.). Soil erosion research methods. Ankeny: Soil and Water Conservation Society, 1988. p. 141-160.

MARQUES, J. J. G. S. M. et al. Índices de erosividade da chuva, perdas de solo e fator erodibilidade para dois solos da região dos cerrados: $1^{a}$ aproximação. Revista Brasileira de Ciência do Solo, Viçosa, v. 21, n. 3, p. 427-434, jul./ set. $1997 \mathrm{a}$.

MARQUES, J. J. G. S. M. et al. Adequação de métodos indiretos para estimativa da erodibilidade de solos com horizonte B textural no Brasil. Revista Brasileira de Ciência do Solo, Viçosa, v. 21, n. 3, p. 447-456, jul./set. $1997 b$.

NIMMO, J. R.; PERKINS, K. S. Aggregate stability and size distribution. In: DANE, J. H.; TOPP, G. C. (Eds.). Methods of soil analysis: physical methods. Madison: Soil Science Society of America, 2002. p. 317-328.

REYNOLDS, W. D.; ELRICK, D. E. A method for simultaneous in situ measurements in the vadose zone of field-saturated hydraulic conductivity, sorptivity, and the conductivity-pressure head relationship. Ground Water Monitoring Review, Washington, DC, v. 6, n. 1, p. 84-95, 1992.

SILVA, A. M. et al. Erosividade da chuva e erodibilidade de cambissolo e latossolo na região de Lavras, sul de Minas Gerais. Revista Brasileira de Ciência do Solo, Viçosa, v. 33, n. 6, p. 1811-1820, dez. 2009.
SILVA, M. L. N. et al. Avaliação de métodos indiretos de determinação da erodibilidade de latossolos brasileiros. Pesquisa Agropecuária Brasileira, Brasília, DF, v. 35, n. 6, p. 1207-1220, jun. 2000.

SILVA, M. L. N. et al. Índices de erosividade das chuvas da região de Goiânia, GO. Pesquisa Agropecuária Brasileira, Brasília, DF, v. 32, n. 10, p. 977-985, out. 1997.

SILVA, M. L. N. et al. Proposição de modelos para estimativa da erodibilidade de latossolos brasileiros. Pesquisa Agropecuária Brasileira, Brasília, DF, v. 34, n. 12, p. 2287-2298, dez. 1999.

WISCHMEIER, W. H. A rainfall erosion index for a universal soil-loss equation. Soil Science Society of America Proceedings, Madison, v. 23, n. 3, p. 246-249, 1959.

WISCHMEIER, W. H. Upslope erosion analysis. In: SHEN, H. W. Environmental impact on rivers. Fort Collins: Colorado State Univiversity, 1972. p. 1-26.

WISCHMEIER, W. H.; MANNERING, J. V. Relation of soil properties to its erodibility. Soil Science Society of America Proceedings, Madison, v. 33, n. 1, p. 131-137, jan./fev. 1969.

WISCHMEIER, W. H.; SMITH, D. D. Predicting rainfall erosion losses from cropland east of the Rocky Mountain. Washington, DC: ARS/USDA, 1965.

WISCHMEIER, W. H.; SMITH, D. D. Predicting rainfall erosion losses: a guide to conservation planning. Washington, DC: USDA, 1978. (Agriculture handbook, 537).

WISCHMEIER, W. H.; SMITH, D. D. Rainfall energy and its relationships to soil loss. Transactions of the American Geophysical Union, Washington, DC, v. 39, n. 2, p. $285-$ 291, 1958. 